\title{
Compact radiation sources based on laser-driven plasma waves
}

D.A. Jaroszynski ${ }^{1, *}$, M.P. Anania ${ }^{1,2}$, C. Aniculaesei ${ }^{1,3}$, G. Battaglia ${ }^{1}$, E. Brunetti ${ }^{1}$, S. Chen $^{1}$, S. Cipiccia $^{1,4}$, B. Ersfeld ${ }^{1}$, D. Reboredo Gil ${ }^{1}$, D.W. Grant ${ }^{1}$, P. Grant ${ }^{1}$, M.S. Hur ${ }^{5}$, L.I. Inigo Gamiz ${ }^{1}$, T. Kang ${ }^{5}$, K. Kokurewicz ${ }^{1}$, A. Kornaszewski ${ }^{1}$, W. Li $^{1}$, A. Maitrallain ${ }^{1}$, G.G. Manahan ${ }^{1}$, A. Noble ${ }^{1}$, L.R. Reid ${ }^{1}$, M. Shahzad ${ }^{1}$, R. Spesyvtsev ${ }^{1}$, A. Subiel ${ }^{1,6}$, M.P. Tooley ${ }^{1}$, G. Vieux ${ }^{1}$, S.M. Wiggins ${ }^{1}$, G.H. Welsh ${ }^{1}$, S.R. Yoffe ${ }^{1}, X$. Yang ${ }^{1,7}$

${ }^{1}$ University of Strathclyde, Glasgow G4 0NG, Scotland, UK, ${ }^{2}$ INFN, Frascati, Italy, ${ }^{3}$ Center for Relativistic Laser Science, IBS, Gwangju 61005, Rep. of Korea. ${ }^{4}$ Diamond Light Source, Oxfordshire OX11 0DE, ${ }^{5}$ School of Natural Science, UNIST, Ulsan, 689-798, Rep. of Korea, ${ }^{6}$ National Physical Laboratory, Teddington, Middlesex, TW11 0LW, UK, ${ }^{7}$ Capital Normal University, and Beijing Advanced Innovation Center for Imaging Technology, Beijing, China

*d.a.jaroszynski@strath.ac.uk

\begin{abstract}
Here we explore ways of transforming laser radiation into incoherent and coherent electromagnetic radiation using laserdriven plasma waves. We present several examples based on the laser wakefield accelerator (LWFA) and show that the electron beam and radiation from the LWFA has several unique characteristics compared with conventional devices. We show that the energy spread can be much smaller than $1 \%$ at $130-150 \mathrm{MeV}$. This makes LWFAs useful tools for scientists undertaking time resolved probing of matter subject to stimuli. They also make excellent imaging tools. We present experimental evidence that ultra-short XUV pulses, as short as $30 \mathrm{fs}$, are produced directly from an undulator driven by a LWFA, due to the electron bunches having a duration of a few femtoseconds. By extending the electron energy to $1 \mathrm{GeV}$, and for 1-2 fs duration pulses of $2 \mathrm{~nm}$ radiation peak powers of several MW per $\mathrm{pC}$ can be produced. The increased charge at higher electron energies will increase the peak power to GW levels, making the LWFA driven synchrotron an extremely useful source with a spectral range extending into the water window. With the reduction in size afforded by using LWFA driven radiation sources, and with the predicted advances in laser stability and repletion rate, ultra-short pulse radiation sources should become more affordable and widely used, which could change the way science is done.
\end{abstract}

Plasma, laser wakefield accelerators, free-electron lasers, high power lasers, radiation sources, femtosecond XUV radiation

\section{INTRODUCTION}

Relativistic plasma wakes are produced by the ponderomotive force of intense laser pulses in plasma, which set in motion electron oscillations at the plasma frequency to create tens-of-micron size accelerating and radiating structures. We explore how high-quality ${ }^{1-4}$, femtosecond ${ }^{5}$ to attosecond ${ }^{6}$ duration, 1-10 kA peak current electron bunches can be accelerated to 0.1-10 GeV energies in millimetres to centimetres by a laser wakefield accelerator (LWFA) driven by a terawatt laser ${ }^{1,7}$. We also show that the un-trapped sheath electrons can lead to unprecedented 10s of nC bunches with energies of 1-5 $\mathrm{MeV}^{8,9}$, which can be transformed into $\mathrm{THz}$ radiation with high efficiency ${ }^{9}$, or used directly in imaging, radiation damage and pulsed radiolysis applications. The rapid development of LWFAs is leading to a paradigm shift in accelerator technology: the size of accelerators can now be reduced by many orders of magnitude compared with conventional technology, simply by taking advantage of space charge fields in plasma. The femtosecond duration bunches from a LWFA ${ }^{5}$ can also be used to produce coherent $\mathrm{THz}$ radiation with high efficiency over a broad spectral range up to $100 \mathrm{THz}$. Since the first proposal to use LWFAs as a compact driver of conventional free-electron lasers (FELs) ${ }^{10}$, excellent progress has been made in this direction, which we describe in this paper. Transverse acceleration in the bubble ${ }^{7,11}$ or an external undulator ${ }^{12,13}$ can produce ultra-short pulses of synchrotron radiation with durations approaching one femtosecond. Coherent transition radiation with characteristics comparable with the far-IR FEL can also be produced simply by the passing the femtosecond duration electron bunches through a thin metal foil or the plasma boundary ${ }^{8,14}$. The coherence of all beam-driven sources 
is governed by the longitudinal spectral density of the electron bunches. The radiation becomes coherent when the bunch is shorter than the wavelength of the emitted radiation, or when a periodic density modulation develops, as in the free-electron laser (FEL) ${ }^{15}$ and ion channel laser (ICL) ${ }^{16}$. In this case bunching occurs at the radiation wavelength due to the ponderomotive force, which leads to coherent enhancement and efficient emission.

\section{THE LASER WAKEFIELD ACCELERATOR}

In the LWFA, electrons are accelerated to high energies by the field of the evacuated plasma "bubble" created by the combination of the ponderomotive force of the laser pulse and the restoring Coulomb force of the ions. In the "bubble regime" electrons form a high density sheath around the bubble, from which electrons are injected when their axial velocity exceeds that of the bubble. When the laser power exceeds the critical power for relativistic self-focussing, the pulse compresses and increases in intensity. This leads to variations in the bubble geometry and velocity, resulting in injection of sheath electrons, which are then accelerated until dephasing. The shortest duration and highest quality bunches are produced close to threshold for self-injection ${ }^{14}$ and, when the perturbations to the bubble velocity/geometry are very

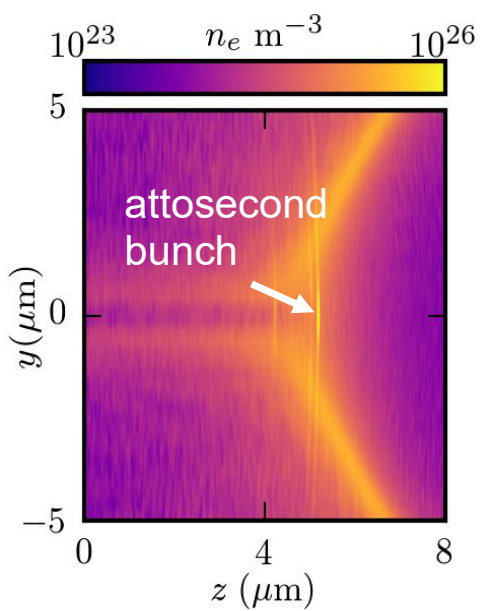

Figure 1: EPOCH PIC simulations of an attosecond bunch being injected at the back of the bubble. brief. In this case, femtosecond ${ }^{5}$ to attosecond ${ }^{6}$ duration electron bunches (see Fig. 1) can be injected into the LWFA bubble.

\section{LWFA EXPERIMENTS}

Experiments have been carried out on the ALPHA beamline ${ }^{7,10}$, where $\approx 1 \mathrm{~J}, 30-40 \mathrm{fs}, 800 \mathrm{~nm}$ laser pulses are focused to

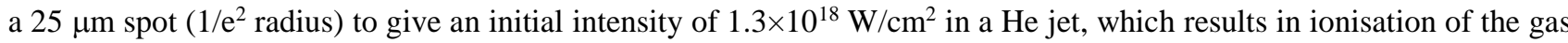
to produce plasma with a density of $1-3 \times 10^{19} \mathrm{~cm}^{-3}$. The normalised vector potential of the laser is $a_{0}=e A / m_{e} c=0.8$, where $A$ is the laser vector potential, $m_{e}$ the electron mass, $e$ the electron charge and $c$ the speed of light. The power is sufficient to trigger relativistic self-focussing, which leads to self-guiding over the full extent of the plasma medium. The main layout of the experiments presented here is shown in Figure 2. Electron spectra are measured using an imaging spectrometer placed $2.6 \mathrm{~m}$ from the LWFA gas jet. Two sets of quadrupole triplets act as a beam transport system. The first (permanent) magnet triplet is situated close to the gas jet (not shown) to collimate the electron beam, and a second (electro-) magnet triplet, Q1-Q3, acts a focusing lens. This is either used to match the beta function to the undulator or optimise beam transport for the electron spectrometer. The undulator is placed $3.5 \mathrm{~m}$ from the LWFA gas jet. When the electron beam is transported through the undulator a small permanent magnet dipole (not shown), with a resolution of around $3 \%$ at $150 \mathrm{MeV}$, is used to measure the electron spectra after the undulator. An XUV spectrometer and X-ray CCD camera are used to measure the undulator radiation spectra and determine the number of photons emitted.

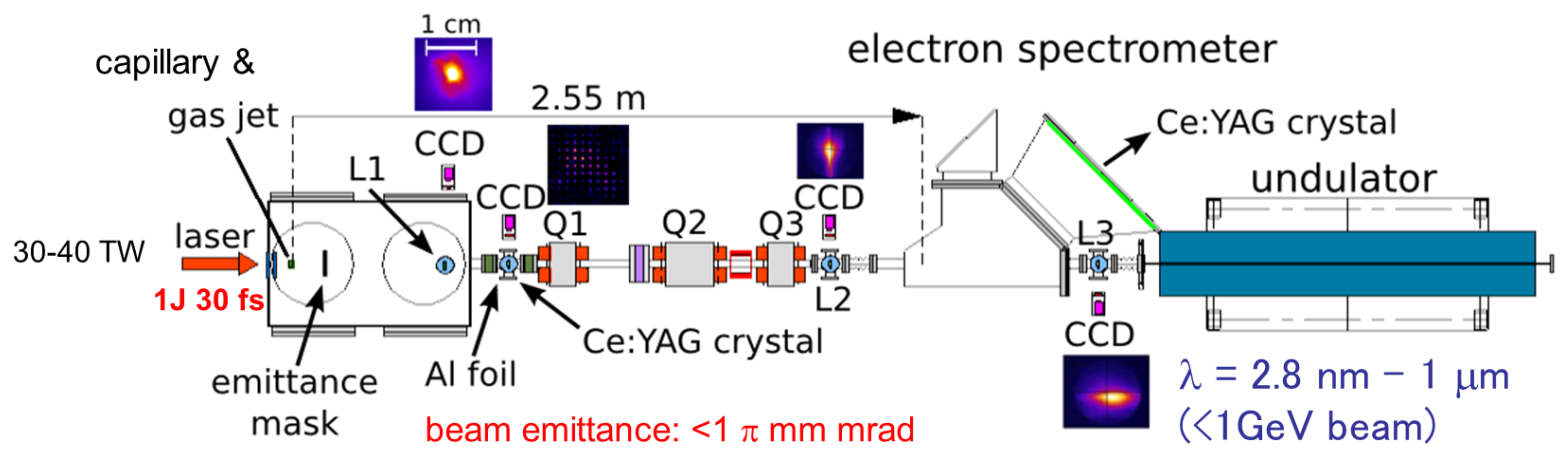

Figure 2. Layout of the ALPHA-X beamline showing the LWFA, quadrupole magnets, dipole spectrometer and 100 period undulator.

In addition to the ALPHA-X measurements we have used 3.5 J pulses from the RAL Gemini laser with a $4 \mathrm{~cm}$ pre-formed plasma channel waveguide ${ }^{7}$ to produce $\mathrm{GeV}$ energy range electron beams ${ }^{11}$. When $a_{0} \geq 1$ relativistic self-focussing increases the intensity and produces a plasma bubble LWFA structure that accelerates electron bunches to energies in the range of $50 \mathrm{MeV}$ to $\mathrm{GeV}$, depending on the plasma density. The LWFA beams are very stable when the laser and plasma are stable (see Fig. 5). The highest quality electron beams are produced close to the threshold for self-injection. Electrons 
that are not injected into the bubble are emitted at $\approx 45$ degrees as high charge, 100s fs duration, MeV energy bunches with a distinct correlation between energy and angle of emission, as shown in Fig. 3, which shows that the slice energy spread can be less than $10 \% .{ }^{8}$

These $10 \mathrm{~s} \mathrm{nC}$ beams are quite divergent and therefore useful for imaging macroscopic objects ${ }^{8}$, as shown in Figure 4. The beams are also efficient emitters of $\mathrm{THz}$ radiation when passed through a thin metal foil or the plasma boundary ${ }^{9}$. Pulses of THz radiation with an energy in excess of several $\mathrm{mJ}$ are emitted with high efficiency using a very simple set up that requires little alignment ${ }^{9}$.

Electron beam energy spectra are measured using an imaging dipole magnet spectrometer and the beam is transported to an undulator using sets of quadrupole magnets.

Beams with an emittance of $1 \pi \mathrm{mm} \mathrm{mrad}^{17}$, energies of $80-300 \mathrm{MeV}^{1,2,4,11}$, and percent level energy spreads $\mathrm{s}^{2,4,18}$, low divergences $(0.5-2 \mathrm{mrad})^{18}$, peak currents $>1 \mathrm{kA}$ and fs bunch duration ${ }^{5}$ have been measured on the ALPHA-X beamline (Figure 2). Theoretical studies (Figure 1) show that attosecond bunches can be produced by perturbing the plasma density to briefly trigger injection ${ }^{6}$.

The smallest energy spread measured for $140 \mathrm{MeV}$ energy beams are around $0.46 \%$ (not de-convoluted for the spectrometer resolution of $0.1 \%$ ), as shown in Figure 5(a). Moreover, the shot-to-shot mean energy variation is below 3\%, as shown in Figure 5 (b), making it possible to undertake experiments in a similar way to using a conventional accelerator. The beam
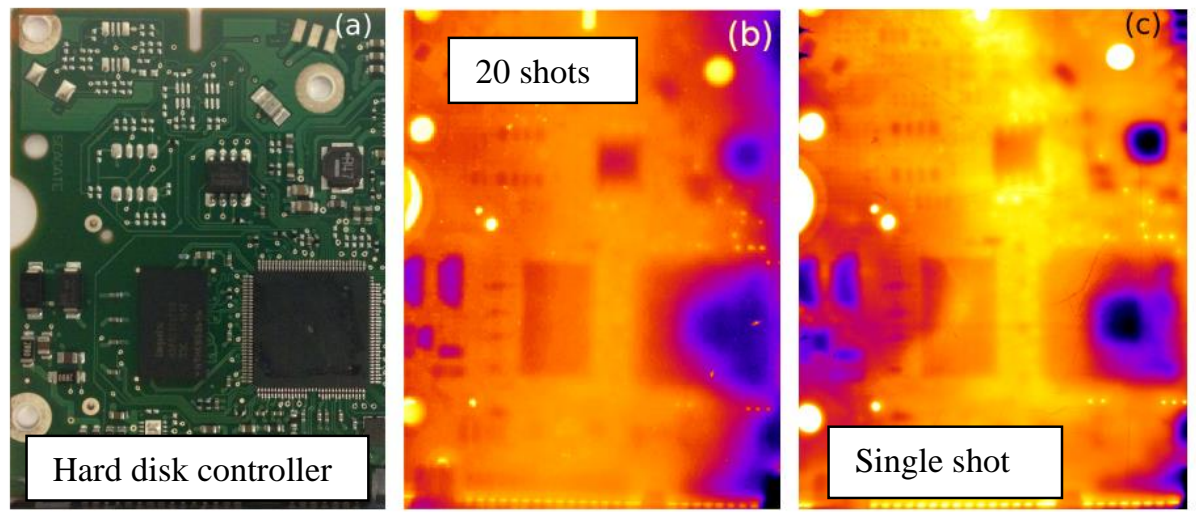

Figure 4. Low energy (1-5 MeV) high charge (10s nC) beams used for direct imaging of a macroscopic object, a hard disk controller (a), showing an image recorded on an image plate after 20 shots (b) and a single shot (c).

divergence at $150 \mathrm{MeV}$ is $\approx 1 \mathrm{mrad}$, for low charge beams $(1-2 \mathrm{pC})^{17}$, and the pointing stability around the same value, as shown in Figure 5 (d). This has enabled an experimental demonstration of undulator radiation using the ALPHA-X ${ }^{7}$ LWFA. Around $95 \%$ of the charge is transmitted through the $6 \mathrm{~mm}$ diameter undulator vacuum chamber, as described below. Although good stability and beam quality of beams in the range of 100-150 MeV can be achieved, it is important to explore whether high quality and stable electron beams can be produced at higher energies. As a first step in this direction we have undertaken experiments using the Rutherford Appleton Laboratory, RAL, Gemini Ti:sapphire laser using 3.5 J, 50 fs laser pulses and a $4 \mathrm{~cm}$ pre-formed plasma capillary waveguide, where we measured mean energies up to $800 \mathrm{MeV}$ and energy spreads of around 4\% (as shown in Figure 5 (c)) close to the spectrometer resolution limit (3\%), indicating that much lower energy spreads are produced ${ }^{11}$. In these experiments we also observed how the plasma bubble acts as a very strong wiggler, producing plasma wiggler (betatron) radiation out to the millionth harmonic, by resonantly multiplying the $1.5 \mathrm{eV}$ laser photons to the gamma-ray spectral region $(7 \mathrm{MeV}){ }^{11}$. 

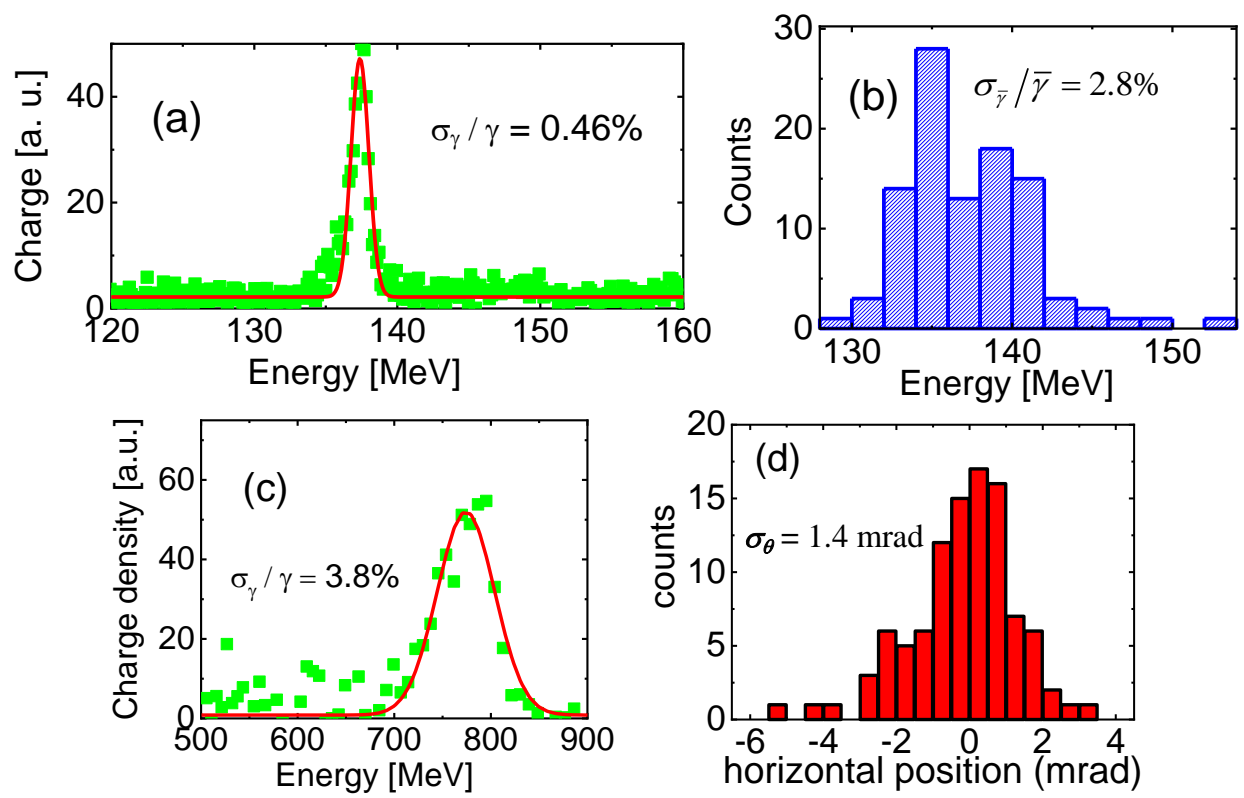

Figure 5: (a) Low energy e-beam energy spectrum with $\sigma_{\gamma} / \gamma=0.46 \%$ from ALPHA-X, (d) corresponding shot-to-shot variation of the mean energy, (c) high energy spectrum of a LWFA driven by $3.5 \mathrm{~J}, 800 \mathrm{~nm}$ laser in a capillary plasma waveguide (measured at Gemini, Rutherford Appleton Laboratory, RAL,), (d) pointing angle distribution (1.4 mrad rms) for a $130 \mathrm{MeV}$ beam.

\section{LWFA DRIVEN UNDULATOR EXPERIMENTS}

The LWFA has excellent pointing stability (Figure 5(d)) when the laser performance is optimised. To demonstrate its suitability as a driver of a synchrotron source, the beam is focussed through an undulator with a deflection parameter $a_{u}=$ 0.38 , number of periods $N_{u}=100$ and periodicity, $\lambda_{u}=1.5 \mathrm{~cm}$, as shown in Figure $2.30 \mathrm{fs}$ pulses of XUV radiation are produced using 120-150 MeV beams, as shown in Fig. 6, which demonstrates the feasibility of a compact, ultra-short pulse XUV synchrotron source ${ }^{13}$. The next stage in these investigations is to increase the energy to $1 \mathrm{GeV}$ and optimise the beam transport to preserve the ultra-short bunch duration in the undulator and investigate FEL operation. This requires a good quality, high peak current beam, which we believe is possible by simply optimising the LWFA and beam transport system.

In these experiments the LWFA is optimised to produce electron bunches of around $150 \mathrm{MeV}$ with charge per bunch of several $\mathrm{pC}$, to ensure the best quality electron beams with a bunch duration of 1-2 fs at source. The undulator radiation spectra for a well optimised beam transport system is measured using a high resolution, imaging XUV spectrometer. The expected wavelength of the emitted undulator radiation is $\lambda=\frac{\lambda_{u}}{2 \gamma^{2}}\left[1+\gamma^{2} \theta^{2}+\frac{a_{u}^{2}}{2}\right] \approx 95 \mathrm{~nm}$, where $\lambda_{u}$ is the undulator wavelength, $\gamma=E / m c^{2}, E$ is the electron energy, $\theta$ is the divergence half-angle. The natural undulator spectral width for a mono-energetic electron beam that is properly matched to the undulator has a typical sync ${ }^{2}$ function shape with a relative spectral width of $\sigma_{\lambda} / \lambda \approx 1 / N_{u}=1 \%$, when the central cone angle $\theta \leq\left(\gamma \sqrt{N_{u}}\right)^{-1} \approx 0.33 \mathrm{mrad}$. The smallest spectral widths are measured for observation angles less than $0.33 \mathrm{mrad}$. The electron energy is maintained around 150 $\mathrm{MeV}$ and electron beam and XUV radiation spectra are measured simultaneously (as shown in Figure 6(a) and 6(b)). For a finite energy spread and electron beam divergence (or collection angle for the XUV spectrometer), $\theta$, the measured undulator spectrum broadens to: $\sigma_{\lambda} / \lambda_{\text {meas. }} \approx\left[\left(2 \sigma_{\gamma} / \gamma\right)^{2}+\left(\sigma_{\theta}{ }^{2} \gamma^{2}\right)^{2}+1 / N_{u}^{2}\right]^{1 / 2}$. Figure 6(b) shows a typical measured XUV spectrum with a width of $\sigma_{\lambda} / \lambda_{\text {meas. }} \approx 2 \%$. The measured electron spectra had a width of $\sigma_{\gamma} / \gamma \approx 3.6 \%$ and a central energy of $140 \mathrm{MeV}$. The mean electron beam energy determined from the undulator radiation spectrum, $E=m c^{2} \sqrt{\frac{\lambda_{u}}{2 \lambda_{\text {meas. }}}\left[1+\gamma^{2} \theta^{2}+\frac{a_{u}^{2}}{2}\right]} \approx 154 \mathrm{MeV}$, is $10 \%$ higher than that of the dipole magnet measurements (Figure 6(a)), 
which indicates a small misalignment. Furthermore, the undulator spectrum (Figure 6(b)) can be used to estimate the upper limit of the electron beam energy spread $\sigma_{\gamma} / \gamma \approx 0.5\left[\left(\sigma_{\lambda} / \lambda\right)_{\text {meas. }}^{2}-\left(\sigma_{\theta}{ }^{2} \gamma^{2}\right)^{2}-1 / N_{u}^{2}\right]^{1 / 2}$, which is $0.85 \%$, neglecting angular spread, and $0.7 \%$, including the broadening due to divergence $(\theta \simeq 0.33 \mathrm{mrad})$. These measurements compare well with the energy spectra shown in Figure 5(a), demonstrating that a high quality electron beam is produced using the LWFA. Moreover, this is much smaller than the energy spectra measured using the low resolution dipole spectrometer. Its resolution can be readily estimated as $\sigma_{\gamma} / \gamma \approx\left[\left(\sigma_{\gamma} / \gamma\right)_{\text {dipole }}^{2}-\left(\sigma_{\gamma} / \gamma\right)_{\text {undulator }}^{2}\right]^{1 / 2} \approx 3.5 \%$, which confirms that these direct measurements are resolution limited. It also emphasises the usefulness of the undulator for non-destructive measurements of the electron energy spread, when the divergence and pointing variations are small (see Figure 5).

The total number of $88 \mathrm{~nm}$ photons emitted from the undulator for a $2 \mathrm{pC}$ electron bunch is around $3 \times 10^{6}$, measured in the central cone defined by the spectrometer slit, in a $30 \mathrm{fs}$ pulse length of (equal to the number of undulator periods). Increasing the electron beam energy to $1 \mathrm{GeV}$ would result in emission of a pulse of $2 \mathrm{~nm}$ photons with a duration equal to that of the electron bunch, i.e. 1-2 fs, assuming an ideal transport system. At higher energies the LWFA produces higher charge electron bunches (up to $100 \mathrm{pC}$ ), with the consequence that between $10^{8}$ and $10^{9}$ photons will be produced per pulse. The peak power of this femtosecond ultra-short XUV pulse would be $10 \mathrm{MW}$ to $1 \mathrm{GW}$. With the development of future high efficiency $1 \mathrm{kHz}$ lasers and increasing the electron energy to $3 \mathrm{GeV}$, the photon rate would be extended to $10^{13}$ photons/s
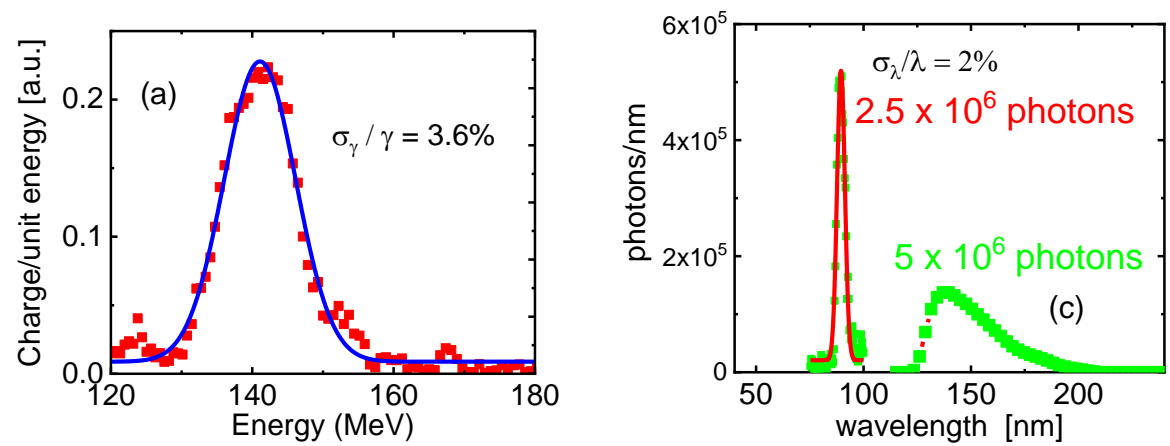

Figure 6 The measured LWFA electron beam energy spread using a low resolution (3\%) spectrometer placed after the undulator. LWFA undulator radiation for different electron beam energies. (a) 100 period $30 \mathrm{fs}$ pulse at $88 \mathrm{~nm}$ with $\sigma_{\lambda} / \lambda \approx 2 \%$, which gives $\sigma_{\gamma} / \gamma \approx 0.8 \%$, (b) peak at $137 \mathrm{~nm}$ showing angular broadened and relatively poor beam transport.

\section{LWFA DRIVEN ION CHANNEL LASER}

The ion-channel laser ${ }^{16}$ is an ultra-compact alternative to the FEL, with the magnetic wiggler replaced by the electrostatic forces of the ion channel, which behaves as an effective compact wiggler. This can be formed by an ultra-short laser pulse or a relativistic bunch of charged particles in plasma. In the ICL, the undulation period depends on the ion density, electron energy and oscillation amplitude, in contrast with the FEL, where it is fixed by the magnet spacing and periodicity (Fig. 7). Amplification in the ICL is analogous to that of an FEL, and is described by scaled FEL-like equations governing the dynamics of the radiation and electron beam ${ }^{16}$. This suggests the feasibility of a compact coherent radiation source for XUV wavelengths, where the gain parameter $\rho$ can be as high as 0.03 , which would give amplification for LWFA electron bunches for realistic energies and emittances ${ }^{16}$. With $\gamma=300,1 \%$ energy spread, $1 \pi \mathrm{mm}$ mrad emittance and high bunch density $n_{b}=10^{20} \mathrm{~cm}^{-3}$ from the LWFA, and a betatron amplitude of $r_{\beta}=$ $10 \mu \mathrm{m}$, the ICL gain parameter $\rho=0.02^{16}$. For these parameters the betatron wavelength $\lambda_{\beta}=\lambda_{p} \sqrt{2 \gamma}$ (equivalent to the undulator period, $\lambda_{u}$ ) for a background plasma density of $1.8 \times 10^{19} \mathrm{~cm}^{-3}$ is $\approx 200 \mu \mathrm{m}$, which gives a gain length of $1.7 \mathrm{~mm}$, and a total saturation length of $\approx 3.5 \mathrm{~cm}^{16}$. This is a very compact coherent XUV source.

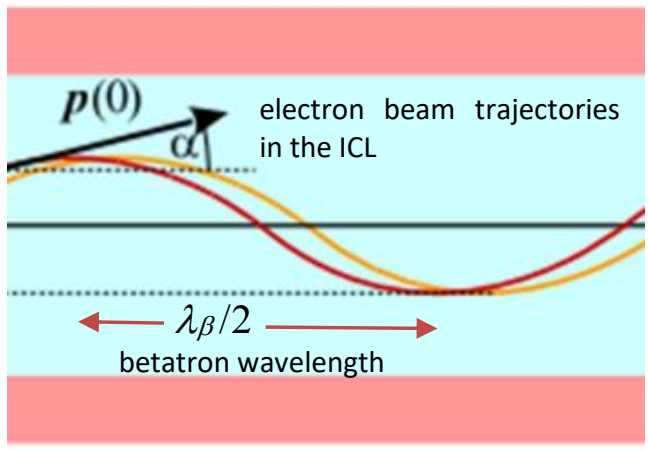

Figure 7: Schematic of the ion channel laser. 


\section{CONCLUSIONS}

We have explored several unique radiation sources based on laser-plasma interactions, some operational and some suggested. These can be quite intense and efficient sources, producing very short duration pulses, down to a single cycle and 100 attosecond duration. Furthermore, with the same laser, a very useful compendium of radiation sources for applications that require tunable ultra-short pulses for pump-probe time resolved studies can be assembled.

\section{ACKNOWLEDGEMENTS}

We acknowledge support of the U.K. EPSRC (grant no. EP/J018171/1, EP/J500094/1 \& EP/N028694/1), the LASERLABEUROPE (grant no. 654148), EuPRAXIA (grant no. 653782), and the Extreme Light Infrastructure (ELI) Project.

Data associated with this paper is available at: http://dx.doi.org/10.15129/b27073ee-e029-4f6b-ae92-1574c2799e5f

\section{REFERENCES}

[1] Mangles, S. P. D. et al., "Monoenergetic beams of relativistic electrons from intense laser-plasma interactions," Nature 431 (7008), 535-538 (2004).

[2] Wiggins, S. M. et al., "High quality electron beams from a laser wakefield accelerator," Plasma Physics and Controlled Fusion 52 (12), (2010).

[3] Wiggins, S. M. et al., "High Quality Electron Beams from a Laser Wakefield Accelerator," 2010 Conference on Lasers and Electro-Optics (CLEO) and Quantum Electronics and Laser Science Conference (QELS) (2010).

[4] Welsh, G. H. et al., "High resolution electron beam measurements on the ALPHA-X laser-plasma wakefield accelerator," Journal of Plasma Physics 78 393-399 (2012).

[5] Islam, M. R. et al., "Near-threshold electron injection in the laser-plasma wakefield accelerator leading to femtosecond bunches," New Journal of Physics 17 (9), (2015).

[6] Tooley, M. P. et al., "Towards Attosecond High-Energy Electron Bunches: Controlling Self-Injection in LaserWakefield Accelerators Through Plasma-Density Modulation," Physical Review Letters 119 (4), (2017).

[7] Jaroszynski, D. A. et al., "Radiation sources based on laser-plasma interactions," Philosophical Transactions of the Royal Society a-Mathematical Physical and Engineering Sciences 364 (1840), 689-710 (2006).

[8] Yang, X. et al., "Three electron beams from a laser-plasma wakefield accelerator and the energy apportioning question," Scientific Reports 7 (2017).

[9] Yang, X., Brunetti, E. \& Jaroszynski, D. A., "High-energy coherent terahertz radiation emitted by wide-angle electron beams from a laser-wakefield accelerator," New Journal of Physics 20 (2018).

[10] Jaroszynski, D. A. \& Vieux, G., "Coherent radiation sources based on laser plasma accelerators," in 10th Workshop on Advanced Accelerator Concepts C. E. Clayton \& P. Muggli, Eds., pp. 902-913, Mandalay Beach, $\mathrm{Ca}(2002)$.

[11] Cipiccia, S. et al., "Gamma-rays from harmonically resonant betatron oscillations in a plasma wake," Nature Physics 7 (11), 867-871 (2011).

[12] Schlenvoigt, H. P. et al., "A compact synchrotron radiation source driven by a laser-plasma wakefield accelerator," Nature Physics 4 (2), 130-133 (2008).

[13] Anania, M. P. et al., "An ultrashort pulse ultra-violet radiation undulator source driven by a laser plasma wakefield accelerator," Applied Physics Letters 104 (26), (2014).

[14] Islam, M. R. et al., "Near-threshold electron injection in the laser-plasma wakefield accelerator leading to femtosecond bunches," New Journal of Physics 17 (2015).

[15] Jaroszynski, D. A., Bakker, R. J., Vandermeer, A. F. G., Oepts, D. \& Vanamersfoort, P. W., "Coherent startup of an infrared free-electron laser," Physical Review Letters 71 (23), 3798-3801 (1993).

[16] Ersfeld, B. et al., "The ion channel free-electron laser with varying betatron amplitude," New Journal of Physics 16 (9), (2014).

[17] Brunetti, E. et al., "Low Emittance, High Brilliance Relativistic Electron Beams from a Laser-Plasma Accelerator," Physical Review Letters 105 (21), (2010).

[18] Jaroszynski, D. A. et al., "Coherent radiation sources based on laser driven plasma waves," Proc. 40th Int. Conf. IRMMW-THz, H2E-1 (2015). 